\title{
Recombinant Extracellular Matrix Protein Fragments Support Human Embryonic Stem Cell Chondrogenesis
}

\author{
Aixin Cheng, MD, PhD, ${ }^{1, *}, \#$ Stuart A. Cain, PhD, ${ }^{1, *}$ Pinyuan Tian, $\mathrm{PhD},{ }^{1}$ Andrew K. Baldwin, PhD,2 \\ Paweena Uppanan, MSc, Cay M. Kielty, PhD, and Susan J. Kimber, PhD ${ }^{1}$
}

We previously developed a 14-day culture protocol under potentially GMP, chemically defined conditions, to generate chondroprogenitors from human embryonic stem cells (hESCs). In vivo work has confirmed the cartilage repair capacity of these cells in a nude rat osteochondral defect model. Aiming to enhance hESC-chondrogenesis, we screened a range of extracellular matrix $(\mathrm{ECM})$ molecules for their ability to support differentiation of hESCs toward chondrocytes. We identified two novel ECM protein fragments that supported hESC-chondrogenesis: Fibronectin III (fibronectin 7-14 protein fragments, including the RGD domain, syndecan-binding domain, and heparin-binding domain) and fibrillin-1 (FBN1) fragment PF8 (encoded by exons 30-38, residues 1238-1605, which contains the RGD motif but not heparin-binding site). These two protein fragments support hESC-chondrogenesis compared with the substrates routinely used previously (a mixture of fibronectin and gelatin) in our directed chondrogenic protocol. We have identified recombinant fibronectin fragment (FN III) and FBNI fragment (PF8) as alternative coating substrates to promote expression of genes known to regulate chondrocytes and code for chondrocyte ECM components. These recombinant protein fragments are likely to have better batch to batch stability than full-length molecules, especially where extracted from tissue/serum.

Keywords: cartilage repair, extracellular matrix, protein fragments, fibronectin, embryonic stem cells

\section{Introduction}

$\mathbf{T}$ he articular cartilage covering of synovial joints disperses loading forces to the underlying subchondral bone and provides a low-friction interface to facilitate joint movement. After the closure of the growth plate, the articular cartilage loses regenerative capacity due to the lack of blood supply. ${ }^{1}$ Chondrocytes, the only cell type in cartilage, have a low proliferation rate and produce all the extracellular matrix (ECM) in the cartilage, mainly proteoglycans and other noncollagenous molecules in adult cartilage, as turnover of the type II collagen network is very slow. ${ }^{2}$ These characteristics contribute to a lack of self-repair capacity in the event of disease or trauma, when the integrity of cartilage is disrupted. ${ }^{3}$

Chondrocytes maintain healthy cartilage and are embedded in the ECM, which contains a number of ECM components. For example, collagen II and collagen XI fibers ${ }^{4-7}$ and proteoglycans with $80 \%$ by weight of the tissue being fluid. ${ }^{8}$ Additional components include a collection of other molecules, such as link protein, COMP, matrilins, decorin, and hyaluronic acid, which act as bridging molecules and by binding to collagens regulate the fibrillogenesis and assembly of the cartilage network. $^{9-12}$ Aggrecan is the major cartilage-specific proteoglycan core protein to which glycosaminoglycan (GAG) chains, predominantly chondroitin sulfate and keratan sulfate, are attached forming a ternary complex with link protein and hyaluronan, ${ }^{13-16}$ and aggrecan also binds to collagens. ${ }^{17}$

The pericellular matrix, the region adjacent to the chondrocytes, has a specialized composition, including collagens and proteoglycans ${ }^{18,19}$ but also fibronectin (FN) and fibrillin $1 .{ }^{20}$ Both interact with collagens, but their location suggests that they are available for interaction with chondrocytes. As far as its role in collagen fibrillogenesis, ${ }^{21,22}$ FN has been shown to stimulate chondrocyte GAG formation in vitro ${ }^{23}$ and has well-established growth factor interactions. ${ }^{24}$ Fibrillin-1 microfibrils are also deposited in the cartilage ECM after adolescence and mutations cause skeletal abnormalities such as overgrowth of long bones. ${ }^{20}$

\footnotetext{
${ }^{1}$ Faculty of Biology, Medicine and Health, University of Manchester, Manchester, United Kingdom.

${ }^{2}$ Academic Group-Engineering, Sports and Sciences, The University of Bolton, Bolton, United Kingdom.

*These authors contributed equally to this work.

\#Current affiliation: Department of Trauma and Orthopaedic, Salford Royal NHS Foundation Trust, Stott Lane, Salford, United Kingdom.
}

(C) Aixin Cheng et al., 2018; Published by Mary Ann Liebert, Inc. This is an Open Access article distributed under the terms of the Creative Commons Attribution License, which permits unrestricted use, distribution, and reproduction in any medium, provided the original work is properly cited. 
$\beta 1$-integrin adhesion molecules are the main ECM receptors for collagens and FN. The functional integrin dimers formed by the alpha and beta transmembrane subunits regulate multiple biological processes such as proliferation, migration, survival, and differentiation, including human embryonic stem cells (hESCs). ${ }^{25,26}$ Collagens interact with cells through $\alpha 1-, \alpha 2-, \alpha 3-$, and $\alpha 10 \beta 1$ receptors and $\alpha 5 \beta 1$ binds to FN. ${ }^{27}$ Specifically, $\alpha 10 \beta 1$ is required for normal development of the cartilaginous growth plates, ${ }^{28}$ while $\alpha \mathrm{V} \beta 6$ is a receptor for fibrillin- ${ }^{29}$ and contains TGF $\beta-1$ binding protein-like modules. ${ }^{30}$

We previously reported a directed differentiation protocol (DDP) for hESCs, which mimics the natural development process toward hyaline cartilage to generate chondrogenic cells. ${ }^{31}$ This protocol exploits the feeder-free and serum-free culture system we developed to provide potential GMP conditions for the growth and expansion of hESCs. ${ }^{26}$ We have shown that hESC-chondrocytes can generate hyaline-like cartilage repair in a nude rat osteochondral-defect model. ${ }^{32}$ Previously, cells have been differentiated on plasma $\mathrm{FN}$ in combination with gelatin. In this study, we used this chemically defined chondrogenic differentiation protocol to screen an array of recombinant ECM molecules and protein fragments, including some found in cartilage, for support of hESC- chondrogenesis. We found FN and fibrillin-1 recombinant fragments support $\mathrm{hESC}$-chondrogenesis in a manner equivalent to full-length FN.

\section{Methods}

\section{Generation and purification of ECM peptide}

All noncommercial protein fragments were expressed in mammalian 293-EBNA cells and modified with either $\mathrm{N}$ or C terminal His-tags. FN fragment FN III (residues 1176-2082 PLS...KKT) was cloned from RNA extracted from ARPE-19 cells and was modified with an N-terminal His6 tag (Fig. 1A). Full-length human FN was cloned from mesenchymal stromal cell (MSC) RNA and had a sequence matching Homo sapiens fibronectin 1 (FN1), transcript variant X9 (XM_005246406.1). This variant contains all FN domains apart from domain EDB (Fig. 1A). The native FN signal sequence was replaced by the BM40 signal peptide and a His6 tag. Recombinant human fibrillin-1 fragments (PF1, PF2, PF8, PF9, PF17-1) and fibrillin-2 fragment (PF17-2) were modified with an N-terminal His6 tag. ${ }^{33-35}$ (Fig. 1B). ADAMTS6 full length is encoded by residues 22-1117, and ADAMTS6 C-terminal region starts at the 1st TSR domain and is encoded by residues 558-1117 modified, with a Cterminal His6 and V5 tag. ${ }^{36}$ Syndecan-4 ectodomain encoded by residues 19-143 (ESI....IFE) 36 and syndecan 2 ectodomain encoded by residues 19-141 (ESR....LFK) were expressed with a C-terminal His10 tag. LTBP-1 was expressed with a C-terminal His6 tag. ${ }^{37}$ Agrin fragments were cloned from RNA extracted from ARPE-19 cells, Agrin K6-SEA (residues 535-1252) and Agrin ST2-LGL3 (residues 1304-2064) were modified with an $\mathrm{N}$-terminal His6 tag. All proteins were purified using immobilized metal ion affinity chromatography (IMAC) (HisTrap FF GE Healthcare), under high salt conditions $(0.5 \mathrm{M} \mathrm{NaCl})$. Fibrillin-1, syndecan-4, and LTBP-1 fragments were further purified using size-exclusion chromatography (Superdex 200 or Superose 6, GE Healthcare). Purity of recombinant proteins was analyzed using SDS-PAGE with Coomassie blue, and protein concentrations were calculated using BCA Protein Assay (Pierce) (Fig. 1C). Full amino acid sequences and expected molecular masses of recombinant protein fragments FN III and PF8, and recombinant full-length FN are shown in Supplementary Figure S1 (Supplementary Data are available online at www.liebertpub.com/tea).

\section{Preparation of ECM matrix assay plates}

A 96-well plate coated with ECM molecules containing, in duplicate, a range of concentrations $(1,10,25 \mu \mathrm{g} / \mathrm{mL})$ fragments of fibrillin-1, fibrillin-2, and agrin as well as cellular and plasma FN was created as previously described. ${ }^{38}$ The plate also contained single concentration of the following ECM proteins: LTBP-1 C-terminal fragment, MAGP-1, syndecan-2 extracellular domain, syndecan-4 extracellular domain, and fibulin 4 . ECM proteins diluted in $80 \mu \mathrm{L}$ PBS were incubated overnight on 96-well clear black plates (Greiner \#655090) at $4^{\circ} \mathrm{C}$. The supernatant was removed, and wells were blocked by the addition of $10 \mathrm{mg} / \mathrm{mL}$ bovine serum albumin (BSA) for $1 \mathrm{~h}$. BSA solution was removed, and the plates were stored at $-80^{\circ} \mathrm{C}$ before use. BSA-coated and uncoated tissue culture plate (TCP) wells were used as controls (Supplementary Table S1).

\section{Quantification of adhered ECM proteins}

To compare the relative quantities of absorbed protein fragments on the tissue culture plastic surface, a modified BCA protein assay was performed (Pierce ${ }^{\mathrm{TM}}$ BCA Protein Assay Kit, 23225). Protein fragments were incubated on 96well tissue culture plastic plates in triplicate overnight at concentrations of 5,10 , and $25 \mu \mathrm{g} / \mathrm{mL}$. The plates were then washed with PBS, before the absorbed protein was resolubilized from the surface using $50 \mu \mathrm{L}$ of $4 \mathrm{M}$ GuanadineHCL for $1 \mathrm{~h}$. Two hundred microliters of BCA working reagent was then added and left to develop for $20 \mathrm{~min}$. Absorbance was measured at $570 \mathrm{~nm}$, and background absorbance was subtracted (PBS only) (Supplementary Fig. S2).

\section{Cell culture and directed chondrogenic differentiation}

MAN6 and MAN7 cells were cultured, as previously described. ${ }^{39}$ In brief, hESCs were cultured on mitomycin Cinactivated mouse embryonic fibroblasts (iMEFs) in hESCmedium. For feeder-free culture, cells were lifted from the iMEF layers with TrypLE, (Thermo Fisher Scientific) and plated onto plasma FN-coated (Millipore, lot 2594323, 2854203) (Supplementary Fig. S3) tissue culture flasks with $\mathrm{mTeSR}^{\mathrm{TM}} 1$ medium (STEMCELL technology). ${ }^{26}$ The hESCs were differentiated with sequential addition of growth factors, as previously described. ${ }^{39}$ In brief, Wnt3a, Activin-A, and BMP2 were applied to differentiate hESCs to primitive streak-mesendoderm (day 1-3), followed by addition of BMP4, follistatin, and GDF5 to differentiate to mesoderm (day 4-8), finally, GDF5 with FGF2 was used to direct chondrogenesis (day 9-14).

\section{Screen novel ECM molecules/protein fragments supporting hESCs chondrogenesis}

hESCs undergoing chondrogenic directed differentiation were seeded onto 96-well TCPs coated with a range of ECM proteins or protein fragments at day 8 of the protocol as $3 \times 10^{4}$ cells $/ \mathrm{cm}^{2}$. Cells were further cultured in chondrogenic medium as described. ${ }^{32}$ On day 14 of the protocol, 


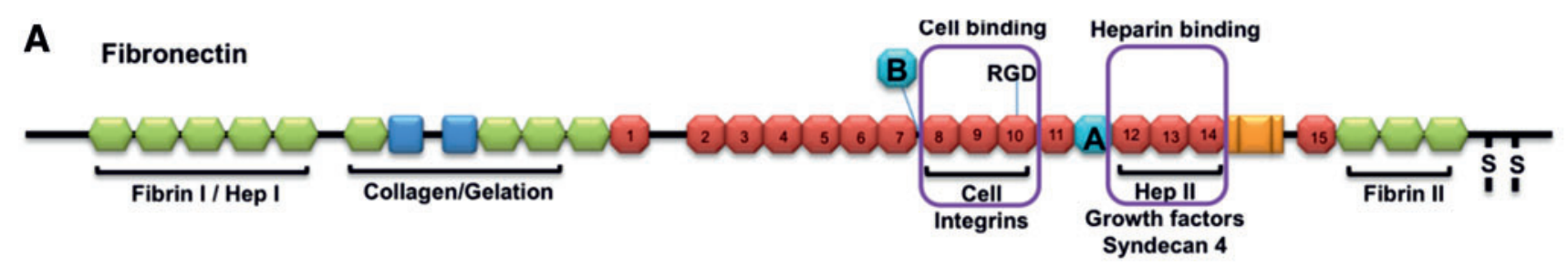

Fn III 7-14
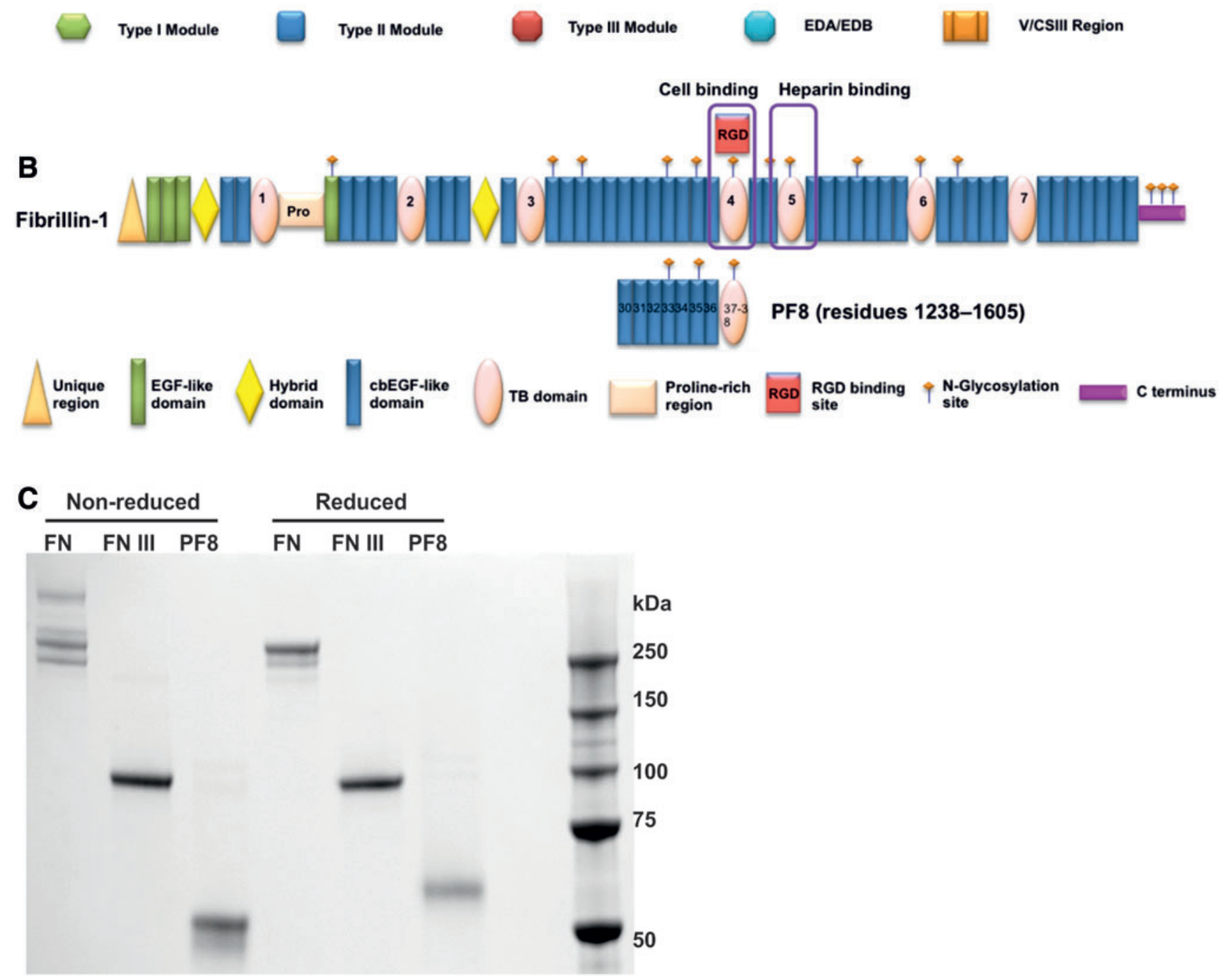

FIG. 1. Schematic diagram of protein fragments. (A) schematic diagram of the domain structure of full-length human fibronectin (FN FL) and fibronectin type III 7 to 14 (FN III) fragment, which contains the RGD-cell binding site and the heparin binding site. (B) domain organization of the recombinant fibrillin-1 fragment PF8 compared with full-length fibrillin-1. (C) SDSPAGE of full-length human fibronectin (FN FL), FN III 7-14 fragment, and fibrillin-1 fragment PF8 run under nonreducing and reducing conditions. Full amino acid sequences and expected molecular masses of FN III, PF8, and recombinant full-length FN are shown in the Supplementary Figure S1. Color images available online at www.liebertpub.com/tea

cells were fixed using $4 \%$ paraformaldehyde followed by Safranin O staining (0.1\% Safranin O, $5 \mathrm{~min})$.

\section{Gene expression analysis}

Total RNA was extracted using an RNeasy ${ }^{\circledR}$ Kit (Qiagen) and DNA removed using Ambion ${ }^{\circledR}$ DNase I (Thermo Sci- entific). Total RNA was reverse transcribed using M-MLV reverse transcriptase (Promega). Expression of candidate genes was determined using SYBR Green PCR Master Mix (Applied Biosystems) with a CFX96/384 Touch $^{\mathrm{TM}}$ Real-Time PCR Detection System (Bio-Rad) using gene-specific oligonucleotide primers (Supplementary Table S2). Expression levels were normalized to $\mathrm{K} \mathrm{KGAPDH}$ and calculated using 
the $2^{-\Delta \mathrm{Ct}}$ method. At least three independent differentiation experiments were performed.

\section{Flow cytometry}

Single-cell suspensions were fixed in ice-cold methanol $\left(10 \mathrm{~min}\right.$ at $-20^{\circ} \mathrm{C}$ ) and permeabilized by incubation with $1 \%$ BSA, $0.5 \%$ triton-X-100 in PBS for 15 min. Cells were incubated with primary antibody (Rabbit mAb anti-SOX9, Cell-Signaling Technology 94794; rabbit anti-Collagen II, $10 \mu \mathrm{g} / \mathrm{mL}$, Novus NB100-91715) diluted in ice-cold blocking buffer (1\% BSA in PBS) overnight at $4^{\circ} \mathrm{C}$ followed by appropriate secondary antibodies. Flow cytometry was conducted using a BD Biosciences Fortessa, and the software Diva gating was used for analysis. FSC-H plotted against FSC-A and a gate drawn around the single cells. This gate was applied to a plot of FSC-A against SSC-A, and then, another gate was drawn around the cells, exclud- ing the debris. The combination of these two gates was applied to the histogram of the fluorescence measurement.

\section{Cell lysate and western blotting}

Cells were washed with cold PBS and lysed in RIPA buffer (Thermo Fisher Scientific 89900) with protease inhibitor cocktail (Roche, 05892970001) for $10 \mathrm{~min}$ on ice, followed by centrifugation at $14,000 \mathrm{~g}$ at $4^{\circ} \mathrm{C}$ for $15 \mathrm{~min}$. The supernatant was saved, $20 \mu \mathrm{g}$ of protein was resolved by $10 \%$ SDS-PAGE gel electrophoresis and transferred to a PVDF membrane blocked with 5\% low-fat milk in TBST for $1 \mathrm{hr}$ followed by primary antibodies (mouse anti-SOX9, $1 \mu \mathrm{g} / \mathrm{mL}$, Abcam 76997; rabbit anti- Collagen II, $10 \mu \mathrm{g} / \mathrm{mL}$, Novus NB100-91715). The membrane was washed three times with PBST and incubated in IRDye 800CM (LI-COR) secondary antibody for $1 \mathrm{~h}$ at room temperature. It was washed with PBST and scanned with the Odyssey ${ }^{\circledR}$ CLx Imaging System.
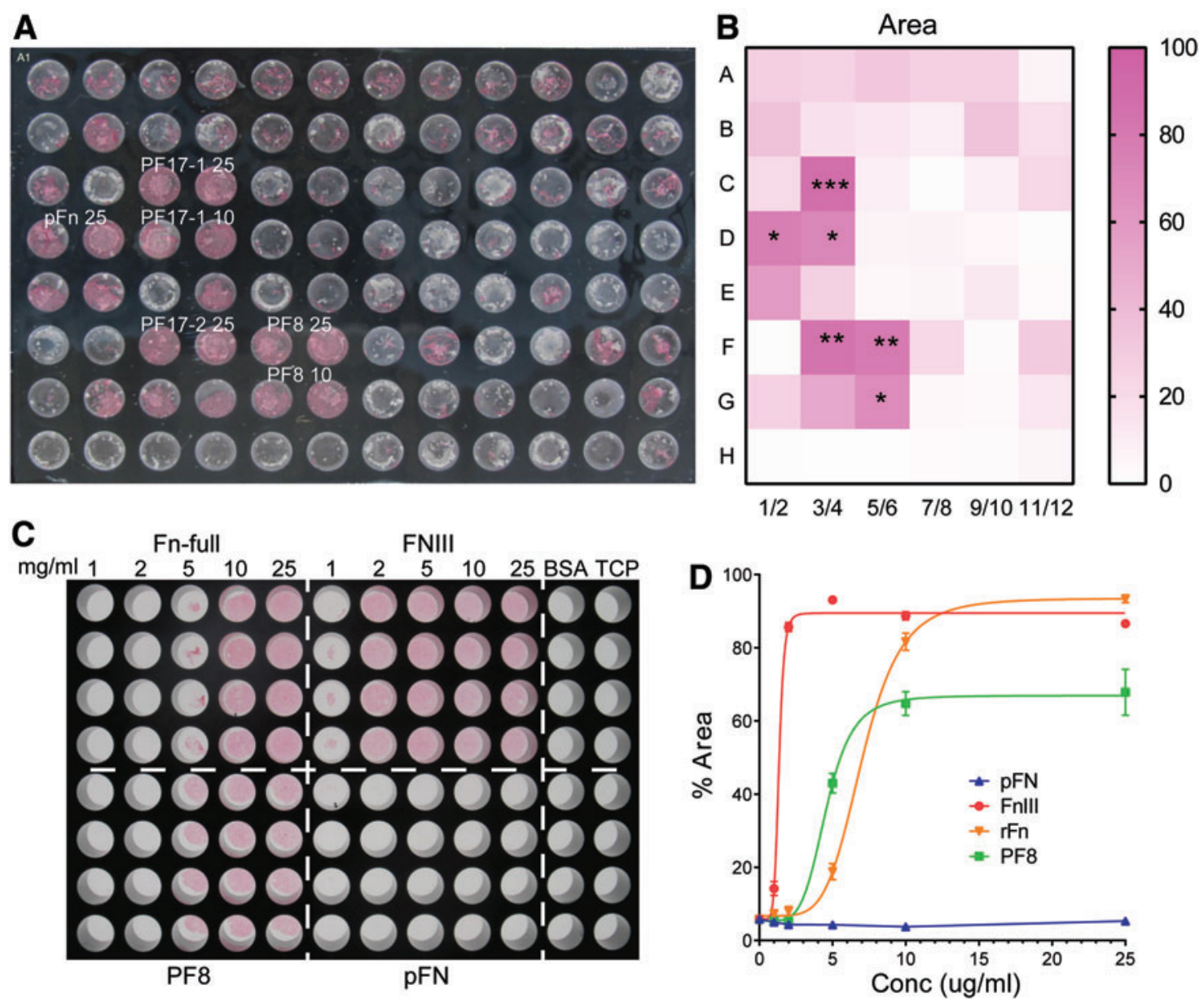

FIG. 2. Screening ECM molecules/peptides supporting hESC-chondrogenesis. On day 8 of DDP, $5 \times 10^{4} \mathrm{cells} / \mathrm{cm}^{2} \mathrm{were}$ seeded on 96-well plates coated with a variety of ECM molecules and further cultured for 6 days in chondrogenic medium. On day 14, cells were fixed and stained with Safranin O (A), (for substrate abbreviations shown in Fig. 2A see Supplementary Table S1), and the staining intensity was quantified with ImageJ (B). (C) Recombinant full-length fibronectin (Fnfull), fibronectin fragment (FN III), fibrillin-1 fragment PF8, and plasma fibronectin (pFN) were plated at increasing concentrations onto a 96-well plate $(n=4)$. Day 8 DDP chondroprogenitors were seeded and cultured until day 14, then stained with Safranin O. The Area of Safranin O staining was quantified and plotted against concentration (D). Nonlinear regression was performed using GraphPad Prism using [Agonist] versus response-Variable slope equation parameters. DDP, directed differentiation protocol; ECM, extracellular matrix; hESC, human embryonic stem cell. Asterisks indicate $P$ values where $* P \leq 0.05 ; * * P \leq 0.01 ; * * * P \leq 0.001$. Color images available online at www.liebertpub.com/tea 


\section{Phalloidin staining}

Cells cultured on different coating substrates were fixed using $4 \%$ paraformaldehyde for $10 \mathrm{~min}$ at room temperature, then quenched and permeabilized, using 0.2 M Glycine $0.5 \%$ Triton x-100. Phalloidin-Atto 565 (Sigma, 94072) staining was performed for 20 min following the manufacturer's instructions and then washed with PBS followed by nuclear staining with DAPI (Thermo Scientific D1306) for $5 \mathrm{~min}$. Images on six-well plates collected on a Leica TCS SP5 AOBS upright confocal using a $20 \times / 0.50 \mathrm{HCX}$ Apo L dipping objective. Images on 96-well plates were collected using CellInsight ${ }^{\mathrm{TM}}$ CX5 High-Content Screening Platform (Thermo Fisher Scientific). Images were then processed and analyzed using Fiji ImageJ (http://imagej.net/Fiji/Downloads). The area covered by the cells for each field of view was calculated by adjusting the threshold to include only the phalloidin stained cells; this gives an indication of the number of cells attached and the amount of cell spreading and was expressed as a\% of the total image area. To quantitate the cell area, phalloidin- and DAPI-stained images were processed using CellProfiler V2.2. ${ }^{40}$ A pipeline was used that identified the positions of the DAPI-stained nuclei, this information was used to identify individual cells on the phalloidin-stained images. The MeasureObjectSizeShape module then calculated cell area and morphology information.

\section{Statistics}

Data are shown as the mean \pm SEM. An unpaired $t$ test for data from two groups or one-way ANOVA for data from groups of three or more was used to compare data. $p$ values $<0.05$ were considered to be significant.

\section{Results}

\section{Screening of ECM proteins/protein fragments supporting $h E S C$-chondrogenesis}

To screen ECM components that support hESCchondrogenesis, a range of ECM proteins and protein fragments was coated onto 96-well TCPs (Fig. 2, Supplementary Table S1). The plates were initially tested for attachment of human MSCs, to ensure sufficient functional protein coating, MSCs were found to adhere and cover a significantly larger cell area, on wells containing FN (cellular and plasma) and on the fibrillin-1 fragments containing the RGD motif (PF17 and PF8) (Supplementary Fig. S4) than on other substrates. An identical plate was then used to screen hESC chondroprogenitors starting at day 8 of the DDP. Cells were seeded at $5 \times 10^{4}$ cells $/ \mathrm{cm}^{2}$ and cultured in chondrogenic medium (containing BMP2, GDF5, and FGF2) for 6 days with medium changed daily. On day 14, cells were fixed and stained with Safranin O to identify GAG formation indicative of chondrogenesis (Fig. 2A). The relative intensity of Safranin $\mathrm{O}$ staining was quantified across the plate using ImageJ (Fig. 2B).

Full-length FN and PF8 (Fig. 1) reproducibly supported generation of Safranin O-positive chondrogenic cells, as did FN fragment FNIII (for structure refer to Fig. 1A) tested separately. We therefore decided to focus on these three molecules for testing further their ability to support hESCs chondrogenesis. To identify the optimal concentration of substrate, increasing concentrations of FN-full, FNIII, PF8, and plasma FN were coated onto a 96-well plate. Day $8 \mathrm{hESC}$ chondroprogenitors were cultured until day 14 , as above mentioned, and stained with Safranin O (Fig. 2C). FN full, FNIII, and PF8 all reach Safranin $\mathrm{O}$ saturation at $10 \mu \mathrm{g} / \mathrm{mL}$, and so, this concentration was chosen for future experiments. The EC50 for
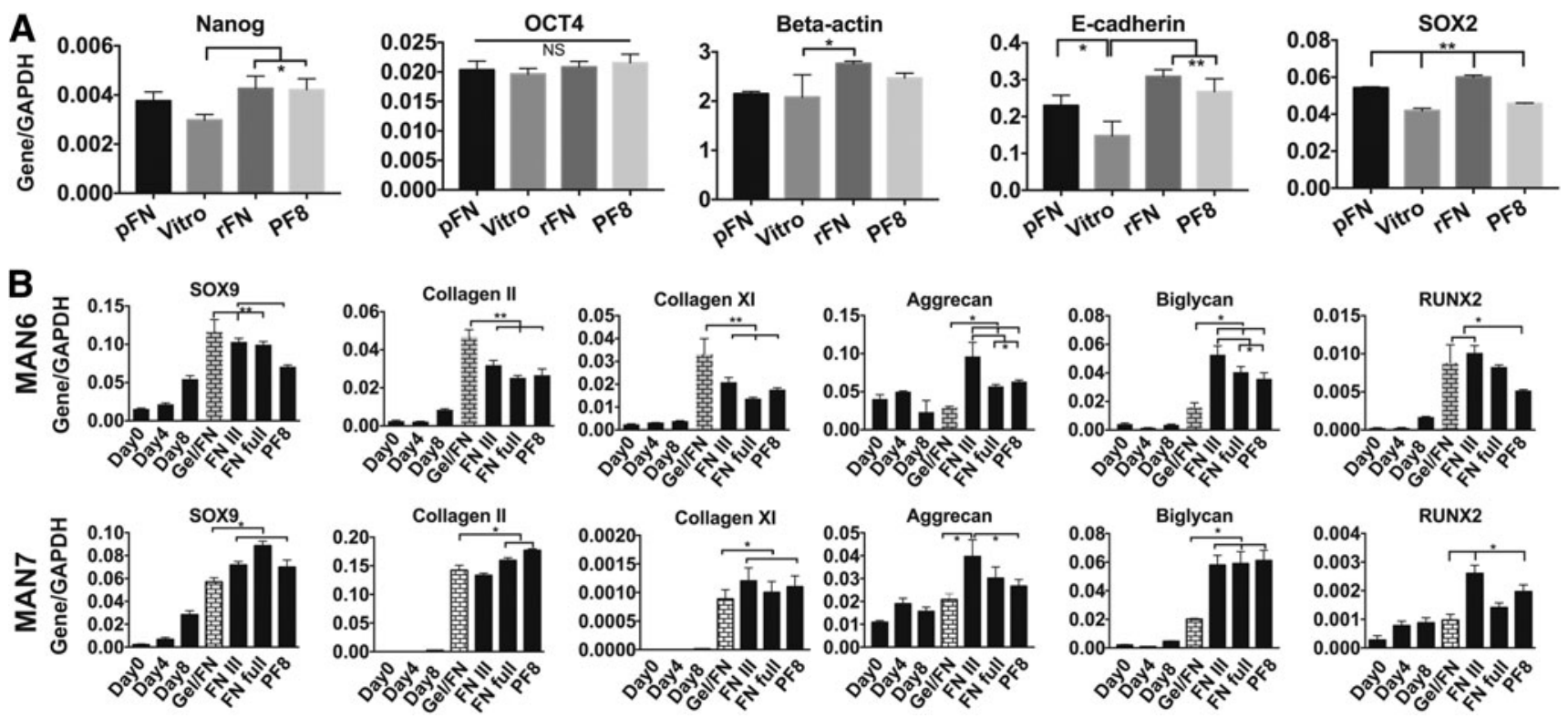

FIG. 3. Recombinant ECM supporting pluripotency and hESCs chondrogenesis. Recombinant full-length FN (rFN), FN fragment (FNIII), and Fibrillin 1 fragment PF8 were tested for supporting pluripotency of MAN7 cells along with plasma FN (pFN) and vitronectin (Vitro). Recombinant FN full length and PF8 maintained pluripotency of MAN7 hESCs after 2 passages (A). Fibronectin rFN, FN III, and PF8 were compared with a mixture of plasma FN and gelatin for supporting hESC-chondrogenesis in MAN6 and MAN7 cell lines; qRT-PCR was used to quantify chondrogenic marker genes (B). Three runs for each cell lines were performed and representative results are shown. Asterisks indicate $P$ values where $* P \leq 0.05$; ** $P \leq 0.01$; NS, non significant. 
saturation were FNII $1.3 \pm 0.04 \mu \mathrm{g} / \mathrm{mL}$, PF8 $4.6 \pm 0.3 \mu \mathrm{g} / \mathrm{Ml}$, and FN full $7.1 \pm 0.2 \mu \mathrm{g} / \mathrm{mL}$. Plasma FN alone did not support chondrogenic staining in these experiments, and so, Gelatin/ Plasma FN mixture $(\mathrm{Gel} / \mathrm{FN})$ was used for further experiments.

\section{Support for hESC-chondrogenesis by identified ECM molecules}

We then tested whether these three substrates could support the culture of the starting hESCs and found that recombinant full-length FN and PF8 support cell adhesion, culture expansion, and maintenance of pluripotent MAN7 cells for two passages, based on gene expression of pluripotency-associated markers to the same extent as plasma FN or vitronectin, used routinely in the laboratory. However, the FNIII fragment did not even support hESC adhesion and was therefore excluded from the hESC qRT-PCR analysis (Fig. 3A).

Next, we evaluated the hESC-chondrogenesis at the transcript level, by applying these substrates from day 8 to day 13 of our DDP with two lines, MAN6 and MAN7. Gene expression analysis showed that even though there was a small variation between the different cell lines, PF8 and FNIII support hESC-chondrogenesis. Compared to 50:50 plasma FN and gelatin used previously, on recombinant full-length $\mathrm{FN}$, the cells expressed equivalent $S O X 9, C O L 2 A 1$, and $C O L 11 A 1$, but higher $A C A N$ and $B G N$. The protein fragments produced similar results, although the increase in $A C A N$ tended to be lower, especially for MAN6 (Fig. 3B). However, RUNX2 express was also higher within cells cultured on full-length FN, FNIII, and PF8 in this line. COL1OA1 was not expressed to any significant extent on any of the substrates (data not shown).

\section{PF8 and FNIII support hESCs chondrogenesis through maintaining SOX9 expression and GAG deposition}

To explore further what underlies the support of PF8 and FNIII protein fragments for hESC-chondrogenesis, we performed western blot analysis for SOX9 protein in cells cultured on the different substrates. We found that using FNIII and PF8 as coating substrate, the expression level of SOX9 was similar as that in the cells cultured on the mixture of FN and gelatin (Fig. 4A, B). To further confirm the protein levels for SOX9 and Collagen II in the cells cultured on different substrates, we performed flow cytometry and found that the expression level of SOX9 and collagen II proteins was very similar in the cells cultured on all of FNIII, PF8, and FN full length, confirming that they can support hESCs chondrogenesis (Fig. 4C). More detailed assessment and quantitation of Safranin $\mathrm{O}$ staining also indicated that PF8, FNIII, and recombinant FN substrates have the potential to promote equivalent or greater deposition of GAG in differentiating chondrogenic cell aggregates compared with the mixture of FN and gelatin. So, these substrates appear to be able to support hESC-chondrocytes ECM deposition (Fig. 5).

To check that there was no significant differences in the amounts of recombinant protein on the tissue culture plastic surface, when applied at $10 \mu \mathrm{g} / \mathrm{mL}$ for the four protein substrates used, the absorption of each protein/protein fragment was also quantified and no significant difference was found, although the amount of protein from FN:gelatin coatings was higher (Supplementary Fig. S2).
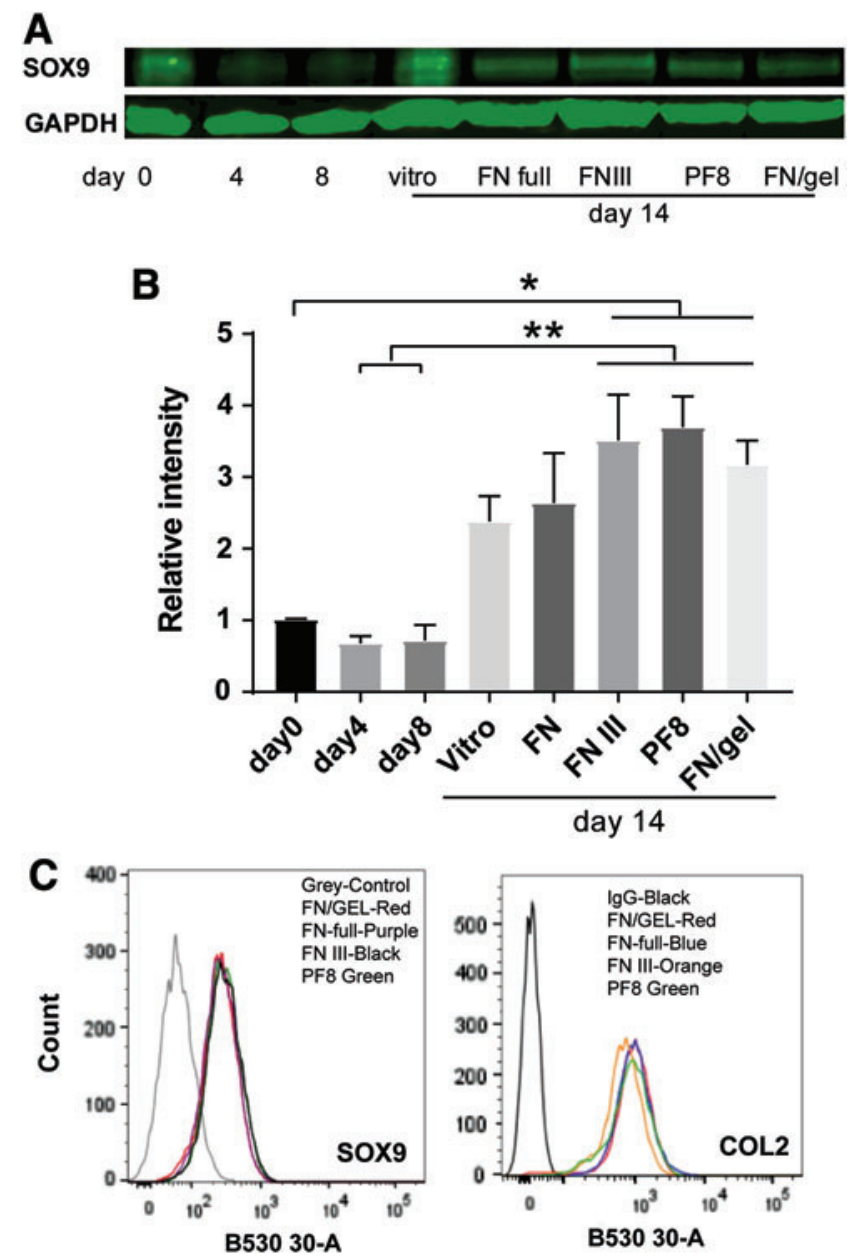

FIG. 4. SOX9 protein level in hESCs during chondrogenesis on different coating substrates. MAN7 hESCs were induced to undergo directed chondrogenic differentiation, on day 8 , different coating substrates were applied and cells were cultured in chondrogenic medium to day 13 . The cell lysates were collected for western blotting to investigate the expression of SOX9. Three independent runs were performed and representative results are shown (A), and the quantification of results from three runs are shown, normalized to the ratio between SOX9 and GAPDH on day 0. Statistical analysis was carried out using GraphPad Prism using 1-way ANOVA and Tukey's multiple comparison test. Asterisks indicate $p$ values where $* p \leq 0.05 ; * * p \leq 0.01$. (B). Flow cytometry analysis of SOX 9 and collagen II level in hESC- chondrogenic cells on different coating substrates. MAN7 hESCs were induced to undergo directed chondrogenic differentiation, on day 8 , different coating substrates were applied and cells were cultured in chondrogenic medium to day 13. The cells were fixed and assessed by flow cytometry using antibodies against SOX9 and Collagen II and Alexa Fluor ${ }^{\circledR} 488$ secondary antibody (C). Color images available online at www.liebertpub.com/tea

\section{Effect of Different Coating Substrates on the Assembly of F-actin}

The chondrocyte cytoskeleton includes actin microfilaments, microtubules, and vimentin-intermediated filaments, ${ }^{41}$ and F-actin is cortical in location. ${ }^{42-45}$ This contrasts with its location in fibroblastic cells and MSCs. We performed 
A

A $\quad \mathrm{Fn} / \mathrm{Ge}$

FNIII

FN-full

PF8

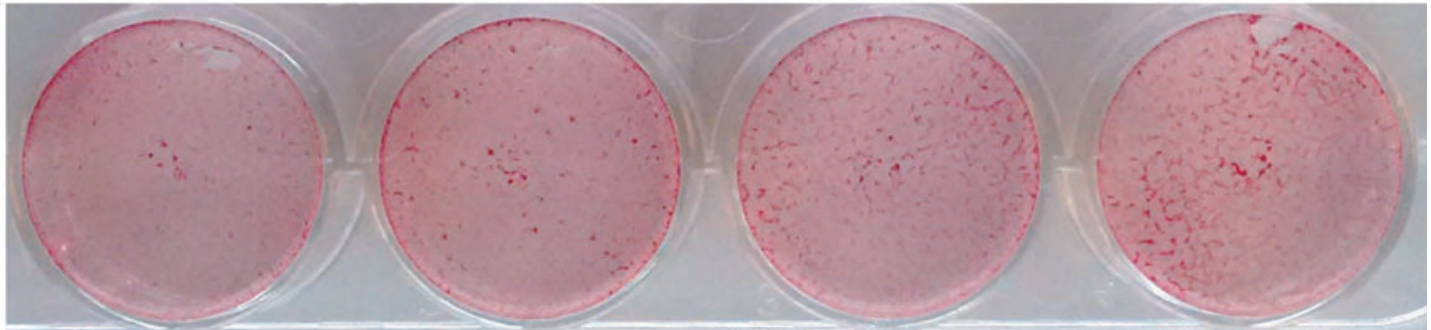

B
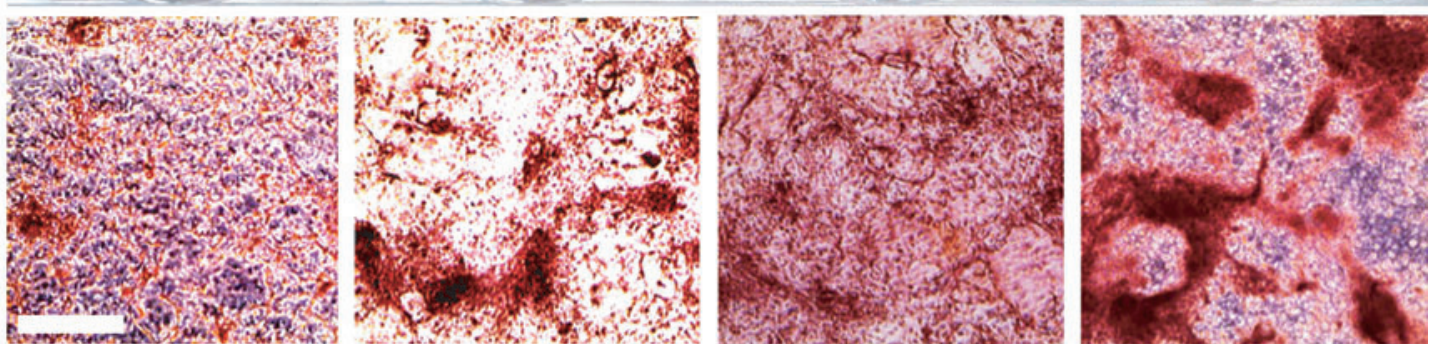

FIG. 5. Safranin $\mathrm{O}$ staining for hESC-chondrogenic cells on different coating substrates. MAN7 hESCs induced to undergo directed chondrogenic differentiation in 12-well plates, on day 8, different coating substrates were applied and cells were cultured in chondrogenic medium to day 13. The cells were fixed and stained with Safranin O, whole plate images were taken (A), and cells were then observed under the microscope (B). Three runs were performed and representative images are shown. Scale bar: $100 \mu \mathrm{m}$. Color images available online at www.liebertpub.com/tea

phalloidin staining at $24 \mathrm{~h}$ after plating on Day 8 , to investigate the distribution of f-actin and found that there was no difference in the distribution of the f-actin on the different substrates, both cortical actin and prominent stress fibers were observed (Fig. 6A). On pFN and PF8, there were significantly fewer cells attached even at higher concentrations of substrate (Fig. 6B and Supplementary Fig. S5), and total cell area was significantly lower for $\mathrm{pFN}$ and PF8 at the saturated concentration of $10 \mu \mathrm{g} / \mathrm{mL}$ (Figs. $2 \mathrm{C}$ and 6C). On PF8 and $\mathrm{pFN}$, cells were less spread indicated by a lower average cell area (Fig. 6D). By day 14, the cytoskeleton had altered, with a loss of stress fibers, and an increase in cortical actin, although no difference was seen between substrates (Fig. 6E). Collagen II deposition could also be detected.

\section{Discussion}

In this study, we exploited our directed hESCs chondrogenic differentiation protocol, mimicking development, ${ }^{39}$ to screen a variety of $2 \mathrm{D}$ coating substrates. Our aim was to find a substrate which could support chondrogenesis to the same extent or better than the plasma FN/gelatin combination used previously. An ideal substrate could be produced as a recombinant protein, in a cost-effective and reproducible manner avoiding batch-to-batch variation. We produced an array of recombinant extracellular proteins and protein fragments, including FN and fibrillin-1 fragments, which were screened for hESC culture expansion and for generation of Safranin $\mathrm{O}$ positive cells in the DDP to generate chondroprogenitors.

Compared with the previously used plasma FN and gelatin mixture, full-length recombinant FN, fibronectin fragment (FNIII), and fibrillin-1 fragment (PF8) (Fig. 1.) could all support adhesion and differentiation of the hESCschondroprogenitors (Figs. 2 and 5). Adding FN to the medium has been shown to promoter MSC chondrogenesis, ${ }^{46}$ and we have shown that plasma FN supports the early stages of hESCchondrogenesis. $^{32,39}$ In this study, we have demonstrated that the FNIII fragment also promotes hESC-chondrogenesis in the later stages of our 2D protocol. Fibrillin-1 (FBN1) is present as a loose meshwork of fibers within cartilage matrix by 20 weeks of fetal gestation and then accumulates in the pericellular matrix forming broad banded fibers by later adolescence. Therefore, we propose that FBN1 may promote the maturation of hESC chondroprogenitors. ${ }^{20}$ Both these protein fragments contain RGD domains, which provide the binding motif for cell surface receptor integrins. ${ }^{47}$ When other FBN fragments that contained homologous EGF repeats, but non-RGDcontaining TB (TGF $\beta$-binding protein-like) domains were used, they did not support cell attachment or chondrogenesis in the initial screen for chondroprogenitors and MSC attachment (Fig. 2 and Supplementary Fig. S4). Similar results for cell attachment of induced pluripotent stem cells to only the RGD-containing protein fragments were seen with an identical 96-well protein array. ${ }^{38}$

It has been shown that interaction of human articular chondrocytes with RGD-containing protein fragments not only initiates cell attachment but also promotes cell migration, proliferation, and maintenance of the chondrogenic phenotype. ${ }^{48}$ So the RGD domain in FNIII and PF8 may function beyond mere adhesion in supporting hESCs chondrogenesis. Indeed, chondrocyte attachment to FN occurs through expression of the major integrin receptor for $\mathrm{FN}$, $\alpha 5 \beta 1$ which interacts with the RGD domain. Notably, $\beta 1$ mediated ECM interaction is reduced in osteoarthritis. ${ }^{49-51}$ It has been suggested that $\mathrm{FN}$-integrin interaction may play a role in chondrocyte survival and retention in the cartilage niche. ${ }^{51}$ Enrichment of the pericellular matrix with FN and its integrin engagement could also contribute to the mechanosensing and mechanoregulation of the chondrocyte. ${ }^{18}$

Previously, we used plasma FN as coating substrates in our differentiation protocol and the batch variation sometimes 

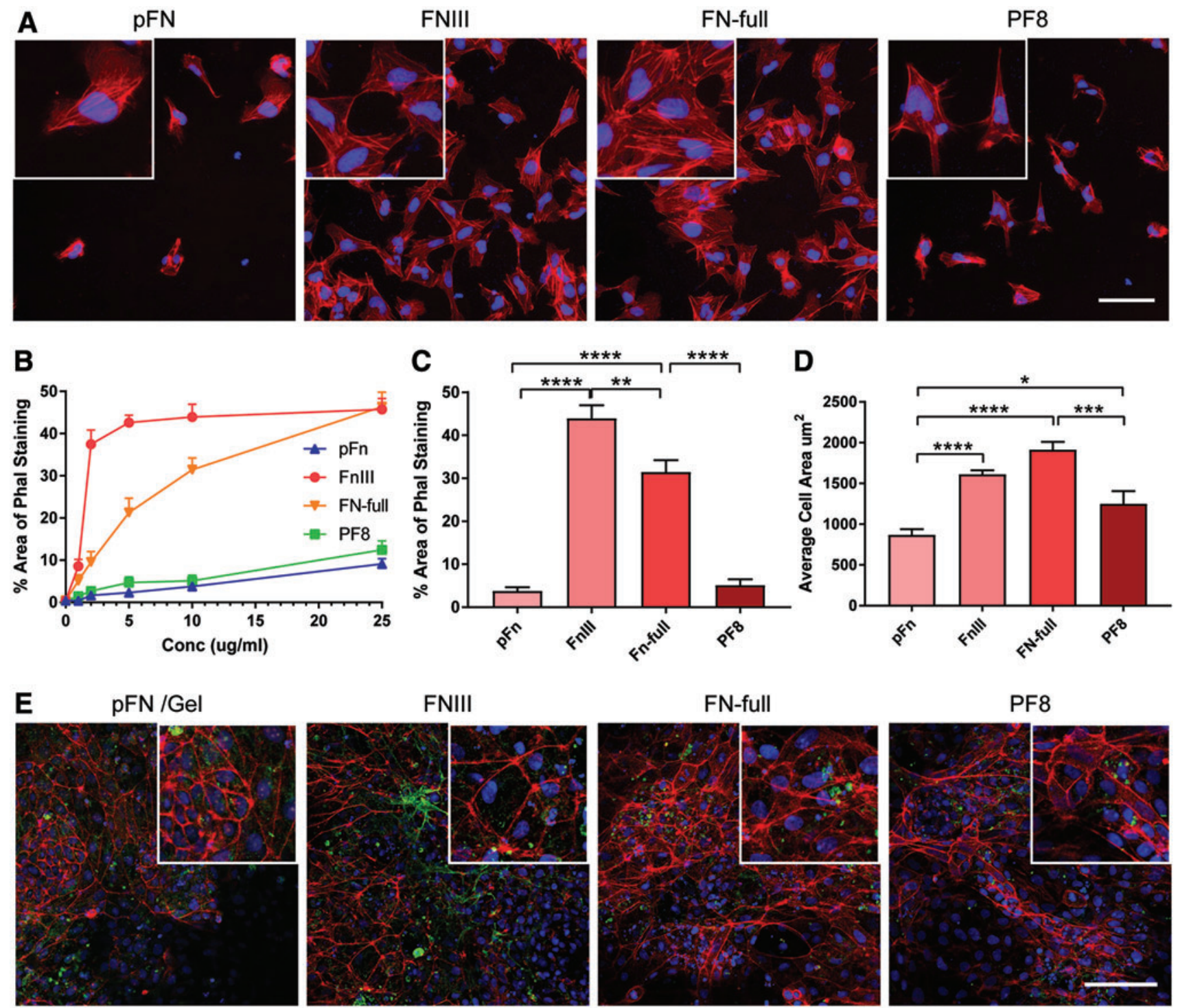

FIG. 6. Imaging of the actin cytoskeleton. Recombinant full-length fibronectin (FN-full), fibronectin fragment (FN III), fibrillin-1 fragment PF8, and plasma fibronectin $(\mathrm{pFN})$ were incubated at increasing concentrations on a 96-well plate $(n=4)$. Day 8 DDP chondroprogenitors were seeded and cultured for $24 \mathrm{~h}$. (A) Adherent cells were visualized with phalloidin and DAPI stains, insert contains expanded region. Images are from $10 \mu \mathrm{g} / \mathrm{mL}$ of substrate. Scale bar: $100 \mu \mathrm{m}$. (B) The average area of phalloidin staining per field of view for increasing concentrations of substrate. (C) The average area of phalloidin staining per field of view and (D) average cell area at $10 \mu \mathrm{g} / \mathrm{mL}$ of substrate were calculated using ImageJ and CellProfiler, respectively. A montage of the images taken and CellProfiler cell identification is shown in Supplementary Figure S5. Statistical analysis was carried out using GraphPad Prism using 1-way ANOVA and Tukey's multiple comparison test. Asterisks indicate $p$ values, where $* p \leq 0.05$; ** $p \leq 0.01$; *** $p \leq 0.001$; **** $p \leq 0.0001$. (E) Day 8 DDP chondroprogenitors were seeded onto different substrates on six-well plates. Day 14 cells were visualized by confocal microscopy using phalloidin (red) and DAPI (Blue) stains. Collagen II (green) was visualized using a mouse anticollagen II antibody (2B1.5, ab185430; Abcam). Insert contains expanded region. Scale bar: $100 \mu \mathrm{m}$. Color images available online at www.liebertpub.com/tea

hindered the reproducibility of the differentiation protocol. The recombinant FN and fibrillin-1 fragment provide more reliable coating substrates for hESCs, which can in future be made under GMP conditions for translational application. Despite some slight variation between the two lines tested in the expression of SOX9, collagen II, and collagen XI, the expression of aggrecan and biglycan transcripts were enhanced by using FNIII and PF8 (Fig. 3). It has been shown that biglycan interacts strongly with collagen type II, but not with type I in vitro. ${ }^{52}$ The enhanced expression of aggrecan and biglycan may indicate that FNIII and PF8 have the capacity to promote cartilage ECM production. The strong Safranin O staining also indicates that FNIII and full-length FN promoted the deposition of GAGs during hESC-chondrogenesis compared with the mixture of plasma FN and gelatin, while plasma FN only supported chondrogenesis in a variable manner. So the transition from FN and gelatin to FNIII or PF8 may be beneficial to promote ECM deposition as well as synthesis. 
SOX9 is the master transcription factor regulating chondrogenesis ${ }^{53}$; if protein fragments can promote the expression of this transcription factor, there is a strong likelihood of their supporting chondrogenesis. Actin polymerization and filament formation has been shown to negatively regulate the transcriptional activity of SOX9 and induce chondrocyte dedifferentiation, in which chondrocytes lose chondrogenic gene expression and cell morphology changes from round to fibroblast-like. ${ }^{54}$ As FN regulates assembly of actin filaments in fibroblasts through the RGD domain and C-terminal heparin-binding region, ${ }^{55}$ we also investigated the effect of different substrates on f-actin assembly. After $24 \mathrm{~h}$ seeding on different coating substrates from Day 8, staining with phalloidin revealed significant cell spreading, but interestingly, fewer hESC-chondrogenic cells attached on PF8 and these were less spread, with a smaller cell area (Fig. 6). However, by day 14, the differences in the chondrogenic cell staining using Safranin O was not as prominent. Moreover, by day 14, imaging of the actin cytoskeleton revealed that it had been remodeled to be mainly cortical in distribution (Fig. 6). As the relaxation of f-actin can potentially restrict the spreading of hESCchondrogenic cells, it could be beneficial to promoting their differentiation as shown for chondrocytes. ${ }^{56}$

When chondrocytes become elongated with flattened and elongated actin stress fibers, they decrease synthesis of type II collagen and aggrecan, but increase type I collagen synthesis, thus, dedifferentiating to a fibroblast-like phenotype. $^{57}$ On the contrary, if chondrocytes are induced to become rounded, the chondrocyte phenotype is rescued. ${ }^{56}$ Taking this together, we propose that FNIII and PF8 may contribute to the promotion of hESC-chondrogenesis through triggering or allowing the remodeling of actin assembly to that more conducive to chondrogenesis.

Our study has demonstrated that the recombinant ECM molecules, FN and its fragment FN III as well as the fibrillin-1 fragment PF8 can replace the mixture of plasma $\mathrm{FN}$ and gelatin as optimal coating substrates for hESCchondrogenesis. As the quality of these recombinant ECM molecules is more reliable than plasma FN, their use will increase the reproducibility of hESC chondrogenic differentiation. Since these substrates can be made under GMP conditions, they are well placed to be used for clinical application of chondrogenic cells for transplantation. Furthermore, the facilitation of cortical actin distribution by these substrates is conducive with a chondrocyte phenotype, and the role of particular protein fragments in this warrants further investigation. Better understanding of the role of the matrix in mesenchymal condensation, formation of the endochondral skeleton, and chondrocyte development to form the permanent cartilage may bring advantages in dissecting the causes of aberrant cartilage and bone formation in chondrodysplasias as well as in generation of chondrocytes for cell therapy.

\section{Acknowledgments}

This work was funded by the UK Regenerative Medicine Platform hub grant MR/K026666/1. We thank Mr Michael Jackson for FACS support. The Faculty of Biology Medicine and Health Bioimaging Facility microscopes were funded by the Biotechnology and Biological Sciences Re- search Council (BBSRC), the Wellcome Trust and the University of Manchester Strategic Fund. PU acknowledges funding from the Royal Thai government.

\section{Disclosure Statement}

No competing financial interests exist.

\section{References}

1. Stempel, J., Fritsch, H., Pfaller, K., and Blumer, M.J. Development of articular cartilage and the metaphyseal growth plate: the localization of TRAP cells, VEGF, and endostatin. J Anat 218, 608, 2011.

2. Verzijl, N., DeGroot, J., Thorpe, S.R., et al. Effect of collagen turnover on the accumulation of advanced glycation end products. J Biol Chem 275, 39027, 2000.

3. Hollander, A.P., Pidoux, I., Reiner, A., Rorabeck, C., Bourne, R., and Poole, A.R. Damage to type II collagen in aging and osteoarthritis starts at the articular surface, originates around chondrocytes, and extends into the cartilage with progressive degeneration. J Clin Invest 96, 2859, 1995.

4. Mendler, M., Eich-Bender, S.G., Vaughan, L., Winterhalter, K.H., and Bruckner, P. Cartilage contains mixed fibrils of collagen types II, IX, and XI. J Cell Biol 108, 191, 1989.

5. Keene, D.R., Oxford, J.T., and Morris, N.P. Ultrastructural localization of collagen types II, IX, and XI in the growth plate of human rib and fetal bovine epiphyseal cartilage: type XI collagen is restricted to thin fibrils. J Histochem Cytochem 43, 967, 1995.

6. Blaschke, U.K., Eikenberry, E.F., Hulmes, D.J., Galla, H.J., and Bruckner, P. Collagen XI nucleates self-assembly and limits lateral growth of cartilage fibrils. J Biol Chem 275, 10370, 2000.

7. Li, Y., Lacerda, D.A., Warman, M.L., et al. A fibrillar collagen gene, Col11a1, is essential for skeletal morphogenesis. Cell 80, 423, 1995.

8. Hardingham, T.E., Fosang, A.J., and Dudhia, J. The structure, function and turnover of aggrecan, the large aggregating proteoglycan from cartilage. Eur J Clin Chem Clin Biochem 32, 249, 1994.

9. Budde, B., Blumbach, K., Ylostalo, J., et al. Altered integration of matrilin-3 into cartilage extracellular matrix in the absence of collagen IX. Mol Cell Biol 25, 10465, 2005.

10. Hedbom, E., and Heinegard, D. Binding of Fibromodulin and Decorin to Separate Sites on Fibrillar Collagens. J Biol Chem 268, 27307, 1993.

11. Hagg, R., Bruckner, P., and Hedbom, E. Cartilage fibrils of mammals are biochemically heterogeneous: differential distribution of decorin and collagen IX. J Cell Biol 142, 285, 1998.

12. Miosge, N., Flachsbart, K., Goetz, W., Schultz, W., Kresse, H., and Herken, R. Light and electron-microscopic immunohistochemical localization of the small proteoglycan core proteins decorin and biglycan in human knee-joint cartilage. Histochem J 26, 939, 1994.

13. Kvist, A.J., Johnson, A.E., Morgelin, M., et al. Chondroitin sulfate perlecan enhances collagen fibril formation. Implications for perlecan chondrodysplasias. J Biol Chem 281, 33127, 2006.

14. Zaucke, F., and Grassel, S. Genetic mouse models for the functional analysis of the perifibrillar components collagen IX, COMP and matrilin-3: implications for growth cartilage 
differentiation and endochondral ossification. Histol Histopathol 24, 1067, 2009.

15. Klatt, A.R., Becker, A.K., Neacsu, C.D., Paulsson, M., and Wagener, R. The matrilins: modulators of extracellular matrix assembly. Int J Biochem Cell Biol 43, 320, 2011.

16. Wilusz, R.E., Sanchez-Adams, J., and Guilak, F. The structure and function of the pericellular matrix of articular cartilage. Matrix Biol 39, 25, 2014.

17. Rojas, F.P., Batista, M.A., Lindburg, C.A., et al. Molecular adhesion between cartilage extracellular matrix macromolecules. Biomacromolecules 15, 772, 2014.

18. Guilak, F., Alexopoulos, L.G., Upton, M.L., et al. The pericellular matrix as a transducer of biomechanical and biochemical signals in articular cartilage. Ann N Y Acad Sci 1068, 498, 2006.

19. Poole, C.A. Articular cartilage chondrons: form, function and failure. J Anat 191 (Pt 1), 1, 1997.

20. Keene, D.R., Jordan, C.D., Reinhardt, D.P., et al. Fibrillin1 in human cartilage: developmental expression and formation of special banded fibers. J Histochem Cytochem 45, 1069, 1997.

21. Kleinman, H.K., Wilkes, C.M., and Martin, G.R. Interaction of fibronectin with collagen fibrils. Biochemistry 20, 2325, 1981.

22. Sevilla, C.A., Dalecki, D., and Hocking, D.C. Regional fibronectin and collagen fibril co-assembly directs cell proliferation and microtissue morphology. PLoS One 8, e77316, 2013.

23. Buckwalter, J.A., and Mankin, H.J. Articular cartilage: tissue design and chondrocyte-matrix interactions. Instr Course Lect 47, 477, 1998.

24. Sawicka, K.M., Seeliger, M., Musaev, T., Macri, L.K., and Clark, R.A. Fibronectin interaction and enhancement of growth factors: importance for wound healing. Adv Wound Care (New Rochelle) 4, 469, 2015.

25. Vitillo, L., Baxter, M., Iskender, B., Whiting, P., and Kimber, S.J. Integrin-associated focal adhesion kinase protects human embryonic stem cells from apoptosis, detachment, and differentiation. Stem Cell Reports 7, 167, 2016.

26. Baxter, M.A., Camarasa, M.V., Bates, N., et al. Analysis of the distinct functions of growth factors and tissue culture substrates necessary for the long-term self-renewal of human embryonic stem cell lines. Stem Cell Res 3, 28, 2009.

27. Hynes, R.O. Integrins: bidirectional, allosteric signaling machines. Cell 110, 673, 2002.

28. Bengtsson, T., Aszodi, A., Nicolae, C., Hunziker, E.B., Lundgren-Akerlund, E., and Fassler, R. Loss of alpha10beta1 integrin expression leads to moderate dysfunction of growth plate chondrocytes. J Cell Sci 118, 929, 2005.

29. Jovanovic, J., Takagi, J., Choulier, L., et al. alpha V beta 6 is a novel receptor for human fibrillin-1 - Comparative studies of molecular determinants underlying integrinRGD affinityand specificity. J Biol Chem 282, 6743, 2007.

30. D'Arrigo, C., Burl, S., Withers, A.P., Dobson, H., Black, C., and Boxer, M. TGF-beta(1) binding protein-like modules of fibrillin-1 and -2 mediate integrin-dependent cell adhesion. Connect Tissue Res 37, 29, 1998.

31. Oldershaw, R., Baxter, M., Brison, D., Hardingham, T., and Kimber, S. The differentiation of human embryonic stem cells towards chondroprogenitors using a chemically defined step-wise protocol. Int J Exp Pathol 90, A71, 2009.
32. Cheng, A., Kapacee, Z., Peng, J., et al. Cartilage repair using human embryonic stem cell-derived chondroprogenitors. Stem Cells Transl Med 3, 1287, 2014.

33. Rock, M.J., Cain, S.A., Freeman, L.J., et al. Molecular basis of elastic fiber formation. Critical interactions and a tropoelastin-fibrillin-1 cross-link. J Biol Chem 279, 23748, 2004.

34. Cain, S.A., McGovern, A., Small, E., et al. Defining Elastic Fiber Interactions by Molecular Fishing: an affinity purification and mass spectrometry approach. Mol Cell Proteomics 8, 2715, 2009.

35. Cain, S.A., McGovern, A., Baldwin, A.K., Baldock, C., and Kielty, C.M. Fibrillin-1 mutations causing weill-marchesani syndrome and acromicric and geleophysic dysplasias disrupt heparan sulfate interactions. PLoS One 7, e48634, 2012.

36. Cain, S.A., Mularczyk, E.J., Singh, M., Massam-Wu, T., and Kielty, C.M. ADAMTS-10 and -6 differentially regulate cellcell junctions and focal adhesions. Sci Rep 6, 35956, 2016.

37. Massam-Wu, T., Chiu, M., Choudhury, R., et al. Assembly of fibrillin microfibrils governs extracellular deposition of latent TGF\{beta\}. J Cell Sci 123, 3006, 2010.

38. Leha, A., Moens, N., Meleckyte, R., et al. A high-content platform to characterise human induced pluripotent stem cell lines. Methods 96, 85, 2016.

39. Oldershaw, R.A., Baxter, M.A., Lowe, E.T., et al. Directed differentiation of human embryonic stem cells toward chondrocytes. Nat Biotechnol 28, 1187, 2010.

40. Carpenter, A.E., Jones, T.R., Lamprecht, M.R., et al. CellProfiler: image analysis software for identifying and quantifying cell phenotypes. Genome Biol 7, R100, 2006.

41. Blain, E.J. Involvement of the cytoskeletal elements in articular cartilage homeostasis and pathology. Int J Exp Pathol 90, 1, 2009.

42. Idowu, B.D., Knight, M.M., Bader, D.L., and Lee, D.A. Confocal analysis of cytoskeletal organisation within isolated chondrocyte sub-populations cultured in agarose. Histochem J 32, 165, 2000.

43. Langelier, E., Suetterlin, R., Hoemann, C.D., Aebi, U., and Buschmann, M.D. The chondrocyte cytoskeleton in mature articular cartilage: structure and distribution of actin, tubulin, and vimentin filaments. J Histochem Cytochem 48, 1307, 2000.

44. Trickey, W.R., Vail, T.P., and Guilak, F. The role of the cytoskeleton in the viscoelastic properties of human articular chondrocytes. J Orthop Res 22, 131, 2004.

45. Blain, E.J., Gilbert, S.J., Hayes, A.J., and Duance, V.C. Disassembly of the vimentin cytoskeleton disrupts articular cartilage chondrocyte homeostasis. Matrix Biol 25, 398, 2006.

46. Singh, P., and Schwarzbauer, J.E. Fibronectin and stem cell differentiation - lessons from chondrogenesis. J Cell Sci 125, 3703, 2012.

47. Ruoslahti, E. RGD and other recognition sequences for integrins. Annu Rev Cell Dev Biol 12, 697, 1996.

48. Vonwil, D., Schuler, M., Barbero, A., et al. An RGDrestricted substrate interface is sufficient for the adhesion, growth and cartilage forming capacity of human chondrocytes. Eur Cell Mater 20, 316, 2010.

49. Kurtis, M.S., Schmidt, T.A., Bugbee, W.D., Loeser, R.F., and Sah, R.L. Integrin-mediated adhesion of human articular chondrocytes to cartilage. Arthritis Rheum 48, 110, 2003.

50. Loeser, R.F. Integrins and chondrocyte-matrix interactions in articular cartilage. Matrix Biol 39, 11, 2014. 
51. Pulai, J.I., Del Carlo, M., Jr., and Loeser, R.F. The alpha5betal integrin provides matrix survival signals for normal and osteoarthritic human articular chondrocytes in vitro. Arthritis Rheum 46, 1528, 2002.

52. Vynios, D.H., Papageorgakopoulou, N., Sazakli, H., and Tsiganos, C.P. The interactions of cartilage proteoglycans with collagens are determined by their structures. Biochimie 83, 899, 2001.

53. Bi, W., Deng, J.M., Zhang, Z., Behringer, R.R., and de Crombrugghe, B. Sox 9 is required for cartilage formation. Nat Genet 22, 85, 1999.

54. Kumar, D., and Lassar, A.B. The transcriptional activity of Sox9 in chondrocytes is regulated by RhoA signaling and actin polymerization. Mol Cell Biol 29, 4262, 2009.

55. Bloom, L., Ingham, K.C., and Hynes, R.O. Fibronectin regulates assembly of actin filaments and focal contacts in cultured cells via the heparin-binding site in repeat III13. Mol Biol Cell 10, 1521, 1999.

56. Loty, S., Forest, N., Boulekbache, H., and Sautier, J.M. Cytochalasin D induces changes in cell shape and promotes in vitro chondrogenesis: a morphological study. Biol Cell 83, 149, 1995.

57. Glowacki, J., Trepman, E., and Folkman, J. Cell shape and phenotypic expression in chondrocytes. Proc Soc Exp Biol Med 172, 93, 1983.

Address correspondence to: Susan J. Kimber, PhD Faculty of Biology, Medicine and Health University of Manchester Michael Smith Building Oxford Road Manchester M13 9PT United Kingdom

E-mail: sue.kimber@manchester.ac.uk

Received: June 7, 2017 Accepted: December 19, 2017 Online Publication Date: February 7, 2018 